\title{
Políticas públicas de descentralización: ¿más gasto y mejores resultados salud?*
}

\section{Decentralizacion public policies: more spending and better health?}

Núm. 6 (2016), pp. 49-65

Blázquez Fernández, Carla*1

Cantarero Prieto, David ${ }^{* 2}$

Pascual Sáez, Marta*3

Recibido: junio, 2015

Aceptado: enero, 2016

JEL Clasif: I7, $\mathrm{H}_{7}$

DOI: $10.5944 /$ reppp.6.2016.14741

*Este trabajo se ha beneficiado de los comentarios y sugerencias de dos revisores, a los que expresamos nuestro agradecimiento. Los posibles errores son responsabilidad exclusiva de los autores.

*1 Carla Blázquez Fernández. Universidad de Cantabria. E-mail: carla.blazquez@unican.es

*2 David Cantarero Prieto. Universidad de Cantabria. E-mail: david.cantarero@unican.es

*3 Marta Pascual Sáez. Universidad de Cantabria. E-mail: marta.pascual@unican.es 


\title{
Resumen
}

En este trabajo analizamos las principales características del sector sanitario español por regiones. En concreto, el estudio muestra las características de dicho gasto así como las diferencias en resultados existentes entre las Comunidades Autónomas de régimen común frente a las forales, una vez que en el año 2002 finalizó el proceso de descentralización sanitaria. Se propone para ello un modelo basado en técnicas de matching, cuantificando las diferencias anteriormente señaladas para el periodo 2002-2013. Los resultados empíricos obtenidos apoyan la hipótesis de efectos asimétricos entre distintos tipos de autonomía regional. Así, el hecho de que una Comunidad Autónoma sea foral (País Vasco y Navarra) está asociado a unos mayores recursos destinados a sanidad, los cuales derivan a su vez en mejores resultados en salud.

Palabras clave: descentralización; gasto sanitario; resultados en salud; técnicas de matching

\begin{abstract}
We investigate the main features of the Spanish regional health care system. The paper points out the main features of health expenditure and the different results between Autonomous Communities in Spain (common regime vs. foral one), once in 2002 the health decentralization process ended. A model based on matching techniques is proposed to analyse these differences for the period 2002-2013. Our empirical results support the hypothesis of asymmetric effects between different types of regional autonomy. Thus, the fact that the Autonomous Community is foral (Basque Country and Navarre) is associated with higher health care resources which derive in better health outcomes.
\end{abstract}

Key Words: decentralization; health expenditure; health outcomes; matching techniques 


\section{Introducción}

Garantizar sistemas sanitarios eficientes, equitativos y solventes es uno de los objetivos clave de cualquier política pública. De esta forma, dado el creciente aumento en el gasto sanitario experimentado en las últimas décadas, repensar su financiación se ha convertido en una cuestión fundamental tanto en España como en el resto de países de su entorno (Urbanos, 2004; Cabasés-Hita, 2006 y 2010; Wendt, 2009; Costa-Font, 2012). Dicha experiencia descentralizadora, se ha caracterizado básicamente por los siguientes elementos: (i) coincidir en el tiempo con un proceso de «envejecimiento del envejecimiento» poblacional; (ii) consolidar la relevancia del sector sanitario dentro de la economía; (iii) estabilizar con el paso del tiempo de la ratio gasto sanitario-PIB; (iv) constatar que el sector público se configure como principal proveedor sanitario.

En España, es la propia Constitución la que recoge el derecho a la protección de la salud. Precisamente, este derecho se ha ido consolidando junto con el cambio de un modelo de Seguridad Social a uno de Sistema Nacional de Salud (SNS, Ley General de Sanidad de 1986). Este modelo se basa pues en el principio fundamental de universalidad (cobertura básica para todos los residentes), siendo actualmente vigente en países como Dinamarca, Finlandia, Irlanda, Suecia, Reino Unido, Grecia, Italia o Portugal. Dicho SNS de prestaciones sanitarias «gratuitas» (Oliva et al., 2014), en su mayoría, en el momento de consumo se combina a su vez con que la atención de la salud esté descentralizada en todas las regiones. Y es que dicho proceso se inició en 1981 y finalizó en 2002, de acuerdo con dos modelos de gestión distintos (Sánchez, Abellán y Oliva, 2013) entre las 17 regiones (excepto Ceuta y Melilla): Comunidades Autónomas Forales y Comunidades Autónomas de Régimen Común.

Si atendemos al gasto sanitario, la mayor parte de la amplia evidencia existente desde Kleiman (1974) y Newhouse (1977) se ha centrado en la importancia de la renta como factor determinante del mismo. Esto es, la cuestión de interés sigue siendo si la elasticidad renta de dicho gasto sanitario es menor o mayor que la unidad. No obstante, el grado de descentralización del país no ha sido siempre considerado en este tipo de estudios (Kanavos y Mossialos, 1996; Di Matteo, 2003), tanto para un solo país como para un grupo de ellos ${ }^{1}$. En todo caso, dicha descentralización sanitaria en cada país puede ser un factor clave en tanto que se relacione con el gasto sanitario final. Dado pues, el carácter asimétrico de esos procesos de descentralización, sus efectos se ven reflejados finalmente en otros indicadores tanto de oferta como de resultados en salud (Jiménez y Smith, 2005; Cantarero, 2006; Blázquez et al., 2013).

Por su parte, respecto a la relación entre descentralización y resultados en salud, señalar que ha sido precisamente en estos últimos años cuando esta cuestión ha cobrado especial interés, si bien la evidencia empírica actual sigue siendo bastante limitada. Y es que el consenso existente en este tipo de literatura muestra que existe un efecto claramente positivo de un mayor grado de descentralización en los principales indicadores objetivos de salud analizados. Además, lo común en muchos estudios es emplear al menos a la esperanza de vida al nacer en términos generales (Mahal et al., 2000; Robalino et al., 2001; Habibi et al., 2003; Jiménez-Rubio y Smith, 2005; Cantarero y Pascual, 2008; Jiménez-Rubio, 2011).

$1 \quad$ A este respecto, cabe destacar los trabajos de Leu (1986), Brown (1987), Barros (1998), Gerdtham et al. (1998), Sen (2005), Baltagi y Moscone (2010), Farag et al. (2012 y 2013), Cantarero y Lago-Peñas (2012), Lago-Peñas et al. (2013) y Thornton y Beilfuss (2016). 
Este artículo pretende pues analizar la evolución y características del gasto sanitario así como sus resultados en salud, prestando especial interés al efecto descentralizador y diferencias existentes entre el grado de autonomía fiscal de las regiones. En concreto, tratamos de discernir si tras la completa descentralización de competencias producida en el año 2002, existen aún diferencias notables entre las Comunidades Autónomas (CC. AA.) de régimen común y las forales (País Vasco y Navarra). Para ello, se utilizan técnicas de «emparejamiento» (matching) que pueden explicar su diferente comportamiento frente a la aproximación tradicional empleada en otros estudios que usan modelos de panel. Así, este trabajo pretende aportar luz sobre cómo esas distintas políticas de gasto tienen a su vez reflejo en los niveles de equidad en el acceso y en la utilización de los servicios sanitarios de dichos sistemas. También, pretende hacer reflexionar acerca de la efectividad real de esas maneras de entender las políticas sanitarias.

Por consiguiente, el trabajo se organiza de la siguiente forma. En el segundo apartado se presenta una contextualización general del gasto y resultados en salud en nuestro país. La tercera sección se refiere a las metodologías así como describe las principales fuentes estadísticas empleadas. A continuación, se presentan los resultados empíricos. Finalmente, se incluye un apartado de conclusiones e implicaciones en términos de planificación sanitaria regional.

\section{Una panorámica del gasto sanitario en España y resultados en salud}

A lo largo de los últimos años se ha apreciado un importante incremento en el volumen de recursos que se dedican a financiar en nuestro país los servicios públicos fundamentales $(\mathrm{SPF})^{2}$, garantes del Estado de Bienestar, en general, y de la sanidad, en particular. En este sentido, la Figura 1, describe la evolución del gasto sanitario público para el periodo más reciente del cual disponemos datos (desde que finalizase el proceso de descentralización), esto es, para el periodo 2002-2012. De igual forma, la Figura 2 muestra el incremento interanual para el mismo periodo y variable. En el mismo, por un lado se observa cómo existe una evolución prácticamente similar en el tiempo tanto en términos per cápita (pc) como en porcentaje con respecto al PIB. Por otro, un intento por preservarlos durante la crisis. Si bien, esto último no ha sido del todo así pues, como puede observarse, ha experimentado cierto retroceso en 2010-2012 al registrar el mismo tasas de crecimiento interanual negativas a raíz de los ajustes presupuestarios abordados, básicamente centrados en el apartado de personal y gastos farmacéuticos (Blázquez et al., 2013; Pérez et al., 2015).

2 Dichos SPF incluyen tres ámbitos: Sanidad, Educación y Protección social (pensiones, desempleo, dependencia y servicios sociales). 


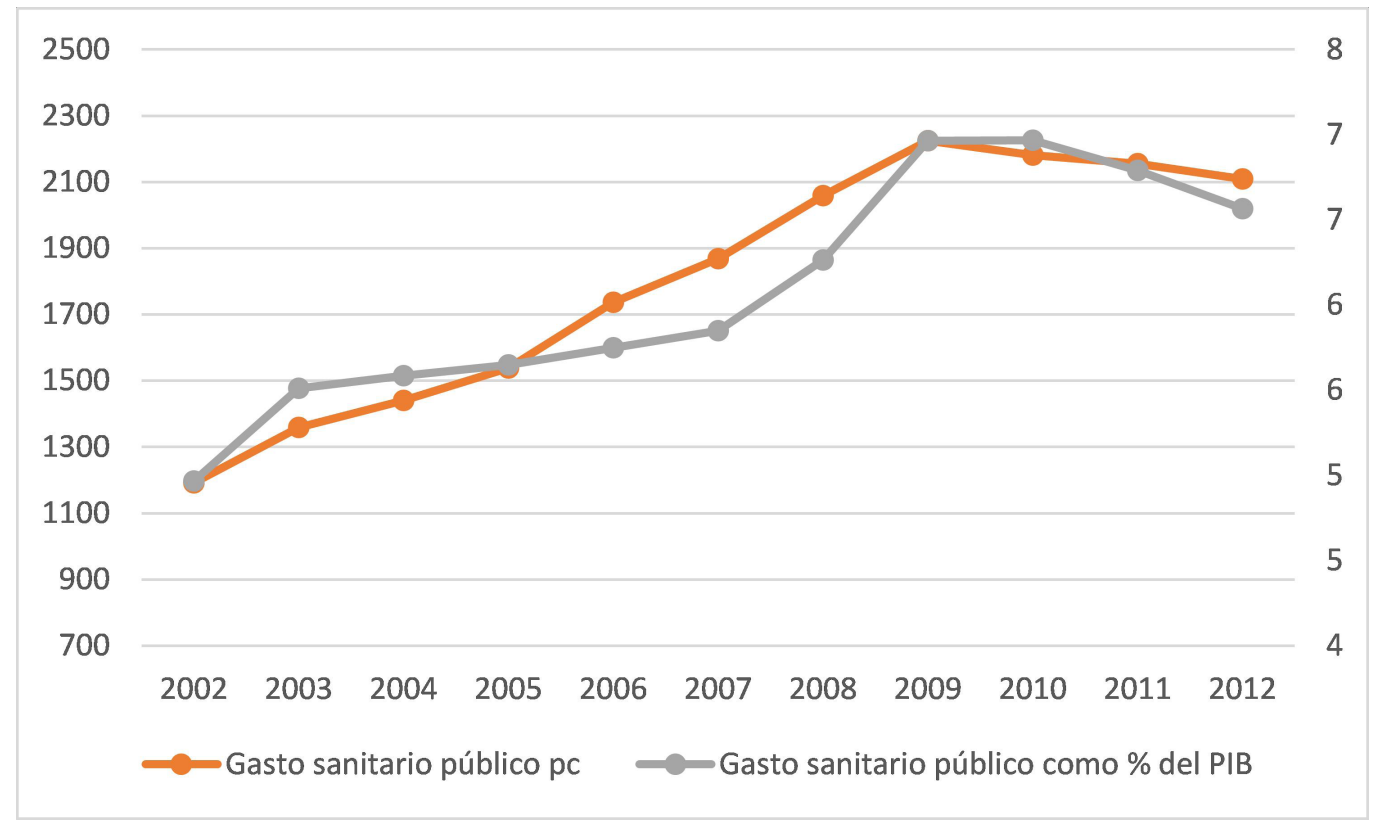

Figura I: Evolución del gasto sanitario público en España. 2002-20I2.

Fuente: elaboración propia a partir de OECD Health Statistics (2015).

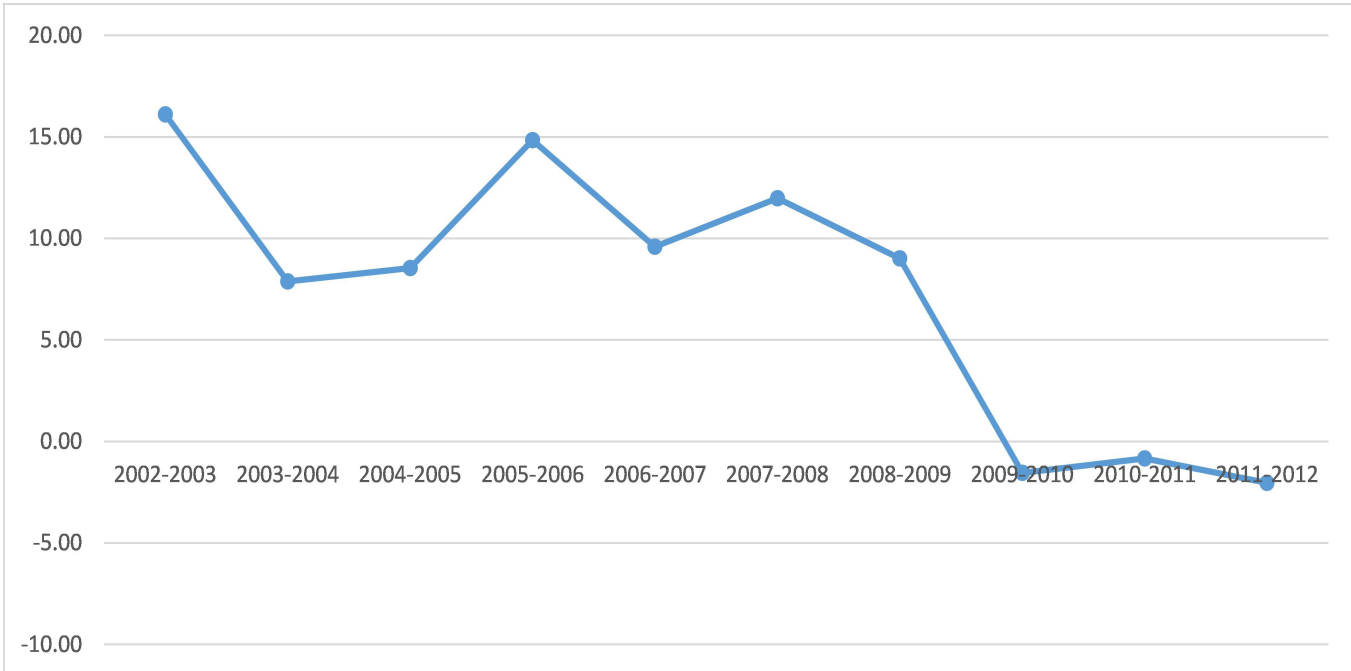

Figura 2: Crecimiento interanual (\%) del gasto sanitario público en España. 2002-20I2

Fuente: elaboración propia a partir de OECD Health Statistics (20I5).

No obstante, a pesar de que desde los diversos ámbitos sean mayores los deseos de una mejor nivelación y de conseguir así una plena igualación del gasto sanitario per cápita entre las regiones españolas, ese indicador presenta considerables diferencias ${ }^{3}$, tal y como se deriva del análisis del Cuadro 1. Según esta información, en 2014 la Comunidad de Andalucía presentaría los niveles más bajos (976,51 euros) seguida a mucha distancia tanto por la Comunidad Valenciana (1073,8 euros) como por las de Madrid (1081,55

3 Si bien esto es consustancial a cualquier proceso de descentralización, pues de lo contrario estaríamos en un modelo plenamente centralizado como el de no hace muchos años con el INSALUD (Repullo, 2007; Blázquez et al., 2014). 
euros) y Baleares (1085,99 euros). En el polo opuesto, encontramos a las Comunidades forales de Navarra y País Vasco (1393,73 y 1570,24 euros, respectivamente) y a Asturias (1398,68 euros) y Cantabria (1336,46 euros).

En este sentido, si bien la descentralización puede ser parte del problema, pensamos que es también parte de la solución tanto a nivel funcional como territorial, pues permite comparar, simular e implementar las mejores políticas sanitarias a modo de «laboratorio de innovaciones en provisión, producción y gestión» (López-Casasnovas y López-Valcárcel, 2015), haciendo de ese modo Benchmarking. Cabe pues señalar que el análisis descriptivo se centra en este punto de manera monográfica en el gasto efectuado, y no en la forma específica de financiación del mismo.

Cuadro I. Gasto público per cápita en salud (euros) de las Comunidades Autónomas: 2007-2014.

\begin{tabular}{|c|c|c|c|c|c|c|c|c|}
\hline $\begin{array}{l}\text { Comunidad } \\
\text { Autónoma }\end{array}$ & 2007 & 2008 & 2009 & 2010 & 2011 & 2012 & 2013 & 2014 \\
\hline Andalucía & 1083,27 & 1166,20 & 1183,50 & 1173,98 & 1113,36 & I I I 4,80 & 1009,75 & 976,51 \\
\hline Aragón & 1261,62 & 1316,45 & 1396,76 & $|4| 6,||$ & $|373,5|$ & 1375,89 & 1210,75 & 1211,20 \\
\hline Asturias & 1245,44 & 1266,86 & 1436,52 & $15 \mid 2,40$ & 1434,77 & 1440,27 & $139 \mid, 67$ & 1398,68 \\
\hline Baleares & 1054,37 & 1116,01 & 1147,98 & 1063,56 & 1056,81 & 1092,05 & 1057,72 & 1082,99 \\
\hline Canarias & 1245,02 & 1350,46 & 1392,53 & 1348,59 & 1194,73 & 1230,18 & 1224,60 & 1238,89 \\
\hline Cantabria & 1259,25 & 1296,56 & 1346,79 & 1343,90 & 1229,73 & 1269,15 & 1310,35 & 1336,46 \\
\hline Castilla y León & 1205,50 & 1303,48 & $1329,7 \mid$ & 1374,73 & 1353,00 & 1347,12 & 1289,14 & $|282,8|$ \\
\hline Castilla La Mancha & 1216,96 & $|289,3|$ & 1370,56 & 1390,84 & 1330,33 & 1228,49 & 1166,53 & 1149,92 \\
\hline Cataluña & 1204,34 & 1243,83 & 1261,00 & 1316,26 & 1220,34 & 1157,18 & 1108,39 & 1102,63 \\
\hline C.Valenciana & 1041,95 & 1084,70 & 1110,91 & 1119,05 & 1077,80 & 1070,81 & 995,39 & 1073,86 \\
\hline Extremadura & 1339,25 & 1457,46 & $1558,|1|$ & 1530,19 & 1417,11 & 1365,75 & 1190,78 & 1204,43 \\
\hline Galicia & 1222,04 & 1307,03 & 1347,28 & 1332,84 & 1268,98 & 1269,43 & 1238,02 & 1201,35 \\
\hline Madrid & 1068,86 & 1108,77 & 1115,16 & 1096,39 & 1099,34 & || $03,7 \mid$ & 1108,63 & 1081,55 \\
\hline Murcia & II 28,64 & $1238,0 \mid$ & 1281,46 & 1357,63 & 1376,43 & 1223,19 & 1071,83 & 1106,64 \\
\hline Navarra & 1332,40 & $|407,6|$ & 1438,26 & 1549,26 & 1513,78 & 1418,95 & 1340,63 & 1393,73 \\
\hline País Vasco & 1365,85 & $15 \mid 4,93$ & 1632,67 & 1666,54 & 1604,97 & $159 \mid, 25$ & 1607,18 & 1570,24 \\
\hline La Rioja & 1336,22 & 1260,09 & 1249,42 & 1276,32 & 1196,58 & 1131,26 & 1090,19 & 1100,40 \\
\hline Promedio & $1212,4 \mid$ & 1278,10 & 1329,33 & $1345,2 \mid$ & 1285,97 & 1260,56 & 1200,68 & 1206,61 \\
\hline $\begin{array}{l}\text { Coeficiente } \\
\text { Variación (\%) }\end{array}$ & 8,62 & 9,21 & 10,96 & 12,36 & 12,29 & 11,56 & 12,99 & $|2,5|$ \\
\hline
\end{tabular}

Fuente: elaboración propia a partir del Ministerio de Sanidad. Recursos económicos del Sistema Nacional de Salud. Presupuestos iniciales (2015).

Todas estas disparidades interregionales, que en muchos casos superan el 30\% respecto a la media, pueden explicarse básicamente por la existencia de factores propios particulares de cada Comunidad Autónoma, así como por cómo opera en cada caso el fenómeno de la «demanda inducida por la oferta». No obstante, las diferencias históricas (statu quo) en financiación y niveles de riqueza pueden jugar también un papel importante (De la Fuente, 2015). 
Por otro lado, la propia inequidad puede entenderse igualmente en términos de desigualdades, lo que conlleva a analizar resultados en salud. En este sentido, en el Cuadro 2 se muestran dichas desigualdades entre Comunidades Autónomas medidas a través de las diferencias en algunos indicadores objetivos comúnmente empleados en la literatura acerca de la salud, como la esperanza de vida al nacer o en buena salud ${ }^{4}$. A este respecto, cabe indicar que esas desigualdades en salud han sido poco atendidas mediante políticas orientadas a reducirlas, existiendo sólo algunas iniciativas destacadas a nivel regional (Borrell et al., 2005; Asociación de Economía de la Salud, 2013; Informe SESPAS, 2014). No obstante, existen excepciones como el Plan de Salud del País Vasco 2013-2020 que incorpora ciertas políticas en ese sentido, así como objetivos cuantitativos para mejorar los resultados y eficiencia de las políticas públicas en términos de equidad, bienestar y salud. Asimismo, el Plan de Salud de Navarra en su versión 2014-2020 avanza de manera análoga.

Cuadro 2. Indicadores de salud: años 2002, 2007 y $201 \mathrm{I}$.

\begin{tabular}{|c|c|c|c|c|c|c|}
\hline \multirow{2}{*}{ Concepto } & \multicolumn{3}{|c|}{$\begin{array}{l}\text { Esperanza de vida al nacer } \\
\text { (años) }\end{array}$} & \multicolumn{3}{|c|}{$\begin{array}{c}\text { Esperanza de vida en buena } \\
\text { salud (años) }\end{array}$} \\
\hline & 2002 & 2007 & 2011 & 2002 & 2007 & 2011 \\
\hline Andalucía & 78,44 & 79,8 & $8 I, 0$ & 51,20 & 63,1 & 65,0 \\
\hline Aragón & 80,23 & 81,7 & 82,6 & 60,30 & 67,1 & 71,2 \\
\hline Asturias & 79,50 & 80,4 & $8 I, 4$ & 50,90 & 58,7 & 63,0 \\
\hline Baleares & 79,40 & 81,7 & 82,2 & 51,40 & 61,7 & 65,6 \\
\hline Canarias & 78,58 & 80,4 & 81,8 & 47,50 & 63,9 & 57,9 \\
\hline Cantabria & 80,21 & 81,1 & 82,6 & 54,70 & 67,8 & 72,0 \\
\hline Castilla y León & 80,18 & 82,1 & 83,4 & 51,30 & 68,7 & 69,6 \\
\hline Castilla La Mancha & 80,94 & 81,5 & 83,0 & 59,50 & 67,1 & 69,5 \\
\hline Cataluña & 79,89 & 81,6 & 82,8 & 56,00 & 60,8 & 66,3 \\
\hline C.Valenciana & 78,89 & 80,6 & 81,9 & 59,30 & 59,4 & 66,3 \\
\hline Extremadura & 79,25 & 80,6 & 81,8 & 52,90 & 63,8 & 67,4 \\
\hline Galicia & 79,88 & 81,0 & 82,2 & 48,60 & 59,1 & 64,2 \\
\hline Madrid & 80,63 & 82,5 & 84,1 & 57,70 & 67,3 & 68,1 \\
\hline Murcia & 78,93 & 80,5 & 82,0 & 54,00 & 60,0 & 65,3 \\
\hline Navarra & 80,87 & 82,5 & 83,8 & 60,20 & 62,8 & 70,5 \\
\hline País Vasco & 80,13 & 81,6 & 82,8 & 59,50 & 63,3 & 68,0 \\
\hline La Rioja & 80,52 & 81,8 & 83,1 & 62,30 & 58,7 & 66,9 \\
\hline Promedio & 79,79 & 81,26 & 82,50 & 55,14 & 63,14 & 66,87 \\
\hline Coeficiente Variación (\%) & 0,98 & 0,97 & $\mathrm{I}, 0 \mathrm{I}$ & 8,31 & 5,45 & 5,11 \\
\hline
\end{tabular}

Fuente: elaboración propia a partir del Ministerio de Sanidad.

4 Dicho concepto de esperanza de vida en salud puede entenderse según el INE como un conjunto diverso de indicadores que tienen en cuenta no sólo la experiencia de mortalidad de la población, sino también la propia morbilidad o incapacidad, pues se define como el promedio de número de años esperados que viva una persona disfrutando de buena salud (en ausencia de limitaciones funcionales o de discapacidad). Ese indicador de años de vida saludable es pues la denominación para la esperanza de vida en buena salud cuando la medida de salud utilizada es la limitación global de actividad y también es conocido como esperanza de vida libre de discapacidad. 


\section{Metodología}

Como se indicó en la sección previa, la literatura académica en Economía de la Salud ha aportado una prolija evidencia empírica acerca de cuáles son los principales determinantes socioeconómicos del gasto sanitario y resultados en salud. En este caso, en nuestro modelo empírico hemos considerado a su vez una nueva estrategia econométrica, como es la estimación de resultados mediante técnicas matching5.

En concreto, vamos analizar los efectos que sobre el gasto sanitario per cápita y los resultados en salud (a través de variables objetivas como la esperanza de vida en buena salud y esperanza de vida al nacer) tiene el hecho de que la Comunidad Autónoma sea foral (y que, por tanto, tenga un mayor grado de autonomía fiscal lo cual le permite poder disponer de más recursos). Esto es, pretendemos cuantificar el efecto causal del «tratamiento» mediante dichas técnicas de emparejamiento o matching. Para ello, necesitamos delimitar dos grupos: un grupo de «tratamiento» y un grupo de control. Resumidamente, nuestro objetivo es analizar el efecto que tiene el hecho de que la comunidad autónoma sea foral en el gasto sanitario y los indicadores/resultados en salud. Esto es, queremos estudiar si dichos resultados son distintos por el hecho de la condición específica de foralidad. A estos efectos, se calculan los correspondientes efectos medios del «tratamiento» («ser foral»).

A efectos de definir formalmente el problema usaremos la notación estándar empleada comúnmente en la literatura relativa a la evaluación de programas públicos. Así, $\mathrm{y}_{1}$ es el resultado de la Comunidad si recibe el «tratamiento» (es País Vasco o Navarra); $\mathrm{y}_{0}$ es el resultado de la Comunidad si no recibe el «tratamiento», mientras que w es un indicador del «tratamiento» recibido, que toma el valor 1 si se recibe el «tratamiento» siendo o en caso contrario. Consecuentemente, el efecto causal del «tratamiento» será: $y_{1}-y_{0}$. El problema aquí es que sólo podemos observar (1)

$$
y=(1-w) y_{0}+w y_{1}=y_{0}+w\left(y_{1}-y_{0}\right) \cdot(1)
$$

Esto implica que no se puede observar el efecto tratamiento individual. Así, nos centraremos entonces en este caso en el análisis de los impactos promedio. En síntesis, vamos a fijarnos especialmente en el efecto medio del tratamiento (ATE) y en el efecto medio sobre los tratados (ATT). Donde:

$$
A T E \equiv E\left(y_{1}-y_{0}\right),(2)
$$

\section{$\mathrm{y}$}

$5 \quad$ Adicionalmente, los datos disponibles en la actualidad, para el caso español, y el grado de originalidad metodológica buscado por los autores llevan a elegir las técnicas de matching frente al panel tradicional (Baltagi y Moscone, 2010). Más allá de una dummy asociada a la foralidad en un panel clásico o aproximación tradicional, en este trabajo ser foral determina el «tratamiento» en las estimaciones efectuadas. Es decir, con el matching construimos grupos de comparación cuando la asignación al tratamiento («foralidad») no es aleatoria pero se realiza según las variables observadas. Por consiguiente, el grupo de comparación es bastante similar al grupo de tratamiento («regiones forales») en términos de los elementos observables antes de que el tratamiento («foralidad») se desarrolle (suponiendo que no existen diferencias en las variables no observadas). 


$$
A T T \equiv E\left(y_{1}-y_{0} \mid w=1\right) \cdot(3)
$$

Dicho efecto medio nos indicará en términos medios el impacto que el hecho que la Comunidad sea foral tiene sobre el gasto sanitario per cápita efectuado y/o los resultados en salud. Este ejercicio se realiza condicionado a un conjunto de variables adicionales. En nuestro caso, en línea con la literatura a este respecto (Baltagi y Moscone, 2010; Cuckler y Sisko, 2013) se considerarán aquí al PIB per cápita en términos reales y a la ratio de dependencia o envejecimiento poblacional.

En este punto, es importante señalar que los datos de gasto sanitario per cápita y de resultados en salud (esperanza de vida) proceden del Ivie-BBVA ${ }^{6}$ y del Ministerio de Sanidad. Por su parte las variables relativas a la renta ${ }^{7}$ (PIB per cápita) y envejecimiento (proporción de personas mayores de 64 años sobre el total) se recogen del Instituto Nacional de Estadística. A este respecto, el Cuadro 3 recoge la definición de dichas variables así como los principales estadísticos descriptivos ${ }^{8}$, contando así con unas 204 observaciones.

Cuadro 3. Definición de variables y estadística descriptiva.

\begin{tabular}{|c|c|c|c|c|c|c|c|c|c|c|c|c|c|}
\hline \multirow[b]{2}{*}{ Variable } & \multirow[b]{2}{*}{ Definición } & \multicolumn{3}{|c|}{ Media } & \multicolumn{3}{|c|}{ Desviación Estándar } & \multicolumn{3}{|c|}{ Mínimo } & \multicolumn{3}{|c|}{ Máximo } \\
\hline & & total & $\begin{array}{c}\text { trata- } \\
\text { miento }\end{array}$ & control & total & $\begin{array}{c}\text { trata- } \\
\text { miento }\end{array}$ & control & total & $\begin{array}{c}\text { trata- } \\
\text { miento }\end{array}$ & control & total & $\begin{array}{c}\text { trata- } \\
\text { miento }\end{array}$ & control \\
\hline gasto & $\begin{array}{l}\text { Gasto sanitario per } \\
\text { cápita (en euros } \\
\text { reales). }\end{array}$ & 1375,47 & 1506,33 & 1358,02 & 207,73 & 188,1769 & 204,42 & 886,95 & 1125,22 & 886,95 & 20384 & 1822,52 & 2038,4 \\
\hline \multirow[t]{2}{*}{$\begin{array}{l}\text { resultados } \\
\text { salud }\end{array}$} & $\begin{array}{l}\text { Esperanza de vida } \\
\text { en buena salud } \\
\text { (años). }\end{array}$ & 61,71 & 64,05 & $6 I, 31$ & 6,22 & 4,36 & 6,56 & 47,5 & 59,5 & 47,5 & 72 & 70,5 & 72 \\
\hline & $\begin{array}{l}\text { Esperanza de vida } \\
\text { al nacer (años). }\end{array}$ & 81,18 & 81,95 & 81,03 & ।,37 & 1,35 & 1,35 & 78,44 & 80,13 & 78,44 & 84,1 & 83,8 & 84,1 \\
\hline renta & $\begin{array}{l}\text { PIB per cápita } \\
\text { (en euros reales). }\end{array}$ & 24023,2 & 30568,4 & 23150,5 & 4782 & 1778,45 & 4360,90 & 14687 & 26986,75 & 14687 & 35182 & 33835,02 & 35182 \\
\hline dep & $\begin{array}{l}\text { Proporción de } \\
\text { personas mayores } \\
\text { de } 64 \text { años sobre } \\
\text { el total. }\end{array}$ & 17,72 & 18,2 & 17,7 & 2,98 & 0,82 & 3,15 & 12,06 & 17,2 & 12,06 & 23,2 & 20,32 & 23,2 \\
\hline foral & $\begin{array}{l}\text { Variable dicotómica. } \\
\text { I si es foral } \\
0 \text { en caso contrario. }\end{array}$ & 0,12 & I & 0 & 0,33 & 0 & 0 & 0 & I & 0 & I & I & 0 \\
\hline
\end{tabular}

Fuente: elaboración propia.

$6 \quad$ En García, et al.(2015) los autores ofrecen datos anuales de gasto público efectivo para el periodo 2002-2013. Precisamente, son esos datos (IVIE- BBVA, 2015) los que usamos en este trabajo.

$7 \quad$ Las variables relativas al gasto sanitario y a la renta, se miden en términos de euros reales.

8 La elección de dichas variables explicativas está restringida principalmente por la falta de información desagregada por regiones para un periodo más reciente en el tiempo. 


\section{Resultados}

Con el matching construimos así grupos de comparación cuando la asignación al tratamiento («foralidad») no es aleatoria (Forales original -final, son siempre las comunidades de País Vasco y Navarra, sin interacciones con otras variables). De este modo, los resultados de las estimaciones efectuadas utilizando Stata 14 se presentan en los Cuadros 4 y 5. En el primero de ellos se muestran los efectos medios del tratamiento (ATE), mientras que en el segundo cuadro se recogen a su vez los correspondientes efectos medios sobre los tratados (ATT).

Si bien es cierto que algunos resultados no resultan claramente significativos en algunos de los años analizados previos a la reciente crisis económica (en dónde, dada la expansión presupuestaria, se acercaron más las situaciones relativas en gasto de regiones de régimen común y forales), se puede comprobar como sí lo son en la mayoría del periodo estimado. Por ello, puede afirmarse que el hecho de que la Comunidad Autónoma sea de régimen foral (es decir, con mayor grado de autonomía fiscal) tiene en términos generales un impacto claramente positivo en el nivel final de gastos sanitarios per cápita.

Por consiguiente, el efecto medio del «tratamiento» de las regiones forales de País Vasco y Navarra supone un incremento en los gastos sanitarios en la media del periodo 2002-2013 de 132,06 euros. Ello representa una clara y notable diferencia sobre el resto de regímenes, que en este caso serían de régimen común. Adicionalmente, podemos señalar cómo el efecto de la condición de foralidad es estadísticamente distinto de cero para País Vasco y Navarra («participantes en el programa»).

En este sentido, podríamos plantearnos si estas diferencias de gasto sanitario per cápita respecto al resto de comunidades de régimen común podrían deberse a ineficiencias en el sistema pues «no siempre gastar más supone gastar mejor» (Pita-Barros, 2006; Cantarero, 2008). Por ello, si atendemos a las estimaciones derivadas de los resultados en salud (los cuales en parte obedecen a los recursos de cada sistema regional de salud), de nuevo, ser una Comunidad foral supondría mejorar claramente dichos resultados ${ }^{9}$ (tanto en términos de esperanza de vida en buena salud como al nacer).

De esta forma, se justificaría igualmente por qué es vital invertir recursos públicos en políticas de salud, así como los efectos positivos asociados a las mismas en regiones con amplia autonomía fiscal. Esto es, el gasto sanitario adicional efectuado por regiones como las forales, donde existe más margen de maniobra financiero que en otras comunidades, sería productivo y rentable socialmente, lo cual contribuye a una mejor rendición de cuentas con transparencia dentro del sector público (Labeaga y Ramiro, 2013).

$9 \quad$ No obstante, los incrementos en la esperanza de vida en buena salud son preferibles a la otra esperanza de vida, dado que reflejan mejor la influencia del sistema sanitario a la hora de alargar la calidad de vida de las personas. En todo caso, cabe señalar que las variaciones en este tipo de indicadores suelen ser lentos en el tiempo en los países desarrollados pues dicha esperanza de vida aumenta en unos tres meses cada año, o lo que es igual, dos años y medio cada década. 
Cuadro 4. Efectos medios del tratamiento (ATE).

\begin{tabular}{|c|c|c|c|c|}
\hline Año & ATE & Error estándar & $\mathbf{z}$ & $\mathbf{p}(\mathbf{z})$ \\
\hline \multicolumn{5}{|c|}{ Gasto sanitario per cápita } \\
\hline 2002 & 127,85 & 41,94 & 3,05 & 0,00 \\
\hline 2003 & || $4,2 \mid$ & 51,07 & 2,24 & 0,03 \\
\hline 2004 & 132,06 & 62,06 & 2,13 & 0,03 \\
\hline 2005 & 106,13 & 57,18 & 1,86 & 0,06 \\
\hline 2006 & 71,92 & 79,99 & 0,90 & 0,37 \\
\hline 2007 & 92,29 & 107,59 & 0,86 & 0,39 \\
\hline 2008 & 130,14 & 58,31 & 2,23 & 0,03 \\
\hline 2009 & 193,98 & 66,39 & 2,92 & 0,00 \\
\hline 2010 & 181,22 & 67,33 & 2,69 & 0,01 \\
\hline 2011 & 172,45 & 65,01 & 2,65 & 0,01 \\
\hline 2012 & 148,89 & 58,29 & 2,55 & 0,01 \\
\hline 2013 & 124,53 & 59,68 & 2,09 & 0,04 \\
\hline Media del periodo $2002-2013$ & 132,06 & 62,06 & 2,13 & 0,03 \\
\hline \multicolumn{5}{|c|}{ Esperanza de vida en buena salud } \\
\hline 2002 & 4,90 & 2,70 & $\mathrm{I}, 82$ & 0,07 \\
\hline 2007 & 0,05 & 2,04 & 0,02 & 0,98 \\
\hline 2011 & 2,95 & 2,14 & 1,37 & 0,17 \\
\hline \multicolumn{5}{|c|}{ Esperanza de vida al nacer } \\
\hline 2002 & 0,50 & 0,45 & $\mathrm{I}, \mathrm{II}$ & 0,27 \\
\hline 2007 & 1,08 & 0,52 & 2,09 & 0,04 \\
\hline 2011 & $\mathrm{I}, 07$ & 0,50 & 2,15 & 0,03 \\
\hline
\end{tabular}

Fuente: elaboración propia. 
Cuadro 5. Efectos medios del tratamiento sobre los tratados (ATT).

\begin{tabular}{|c|c|c|c|c|}
\hline Año & ATT & Error estándar & $\mathbf{z}$ & $\mathrm{p}(\mathrm{z})$ \\
\hline \multicolumn{5}{|c|}{ Gasto sanitario per cápita } \\
\hline 2002 & $1 \mid 4,43$ & 12,10 & 9,46 & 0,00 \\
\hline 2003 & 96,85 & 12,86 & 7,53 & 0,00 \\
\hline 2004 & 162,48 & 18,96 & 8,57 & 0,00 \\
\hline 2005 & 177,03 & 14,19 & 12,48 & 0,00 \\
\hline 2006 & $|29,5|$ & 10,06 & 12,87 & 0,00 \\
\hline 2007 & 148,42 & 28,67 & 5,18 & 0,00 \\
\hline 2008 & 174,14 & 37,13 & 4,69 & 0,00 \\
\hline 2009 & 224,03 & 30,95 & 7,24 & 0,00 \\
\hline 2010 & 170,73 & 10,92 & 15,63 & 0,00 \\
\hline 2011 & $|84,1|$ & 41,09 & 4,48 & 0,00 \\
\hline 2012 & 177,33 & 6,86 & 25,85 & 0,00 \\
\hline 2013 & 167,08 & 7,53 & 22,19 & 0,00 \\
\hline Media del periodo $2002-2013$ & 162,48 & 18,96 & 8,57 & 0,00 \\
\hline \multicolumn{5}{|c|}{ Esperanza de vida en buena salud } \\
\hline 2002 & 3,85 & 0,30 & 12,70 & 0,00 \\
\hline 2007 & 2,25 & 0,22 & 10,39 & 0,00 \\
\hline 2011 & 0,50 & 2,62 & 0,19 & 0,85 \\
\hline \multicolumn{5}{|c|}{ Esperanza de vida al nacer } \\
\hline 2002 & 0,61 & 0,32 & 1,90 & 0,06 \\
\hline 2007 & 0,45 & 0,39 & 1,15 & 0,25 \\
\hline 2011 & 0,60 & 0,28 & 2,12 & 0,04 \\
\hline
\end{tabular}

Fuente: elaboración propia. 


\section{Conclusiones}

A lo largo de los últimos años se ha producido en España (al igual que en otros países con similares características) un importante incremento del gasto sanitario público. Dicho fenómeno, ha suscitado una gran preocupación por conocer sus determinantes y características, dada la importancia de garantizar la solvencia del sistema sanitario en un contexto europeo general de consolidación fiscal. Adicionalmente, dos son las peculiaridades esenciales de dicho modelo.

En primer lugar, su cobertura prácticamente «universal», a pesar de que es cierto que varias de las últimas reformas estructurales han supuesto limitar dicho acceso a la prestación a determinados colectivos. En segundo lugar, su carácter descentralizado (de manera completa y efectiva a partir del año 2002). Así, si bien es cierto que la sanidad es competencia de las Comunidades Autónomas, entre las mismas existen dos grupos claramente diferenciados en cuanto a su grado de autonomía fiscal: las de régimen común y las forales (País Vasco y Navarra).

De esta forma, este trabajo ha tratado de analizar si existen diferencias en el gasto sanitario per cápita entre esos distintos tipos de Comunidad así como los resultados en salud asociados al mismo. Para ello, se han considerado los datos más recientemente disponibles desde que comenzó el proceso de descentralización sanitaria regional en todo el país. Al igual que fuera demostrado en otros estudios previos para el caso español (CostaFont y Pons-Novell, 2007; Cantarero y Lago-Peñas, 2010 and 2012), los resultados empíricos obtenidos apoyan la importancia de la condición de foralidad en términos de gasto y/o resultados sanitarios. Así, en este trabajo nuestros hallazgos muestran cómo el hecho de que la Comunidad Autónoma sea foral incrementa notablemente el gasto sanitario per cápita realizado en todo el periodo analizado, dada su mayor capacidad para obtener recursos a la hora de financiar dicho gasto debido a su prácticamente plena autonomía fiscal. Asimismo, los resultados (output) en salud también serían más favorables para dichas comunidades forales frente al resto del país. Por lo cual, entendemos que dicho gasto adicional sería entonces productivo y rentable socialmente. Esto es, serían regiones que dedicando más recursos a la sanidad pública conseguirían mejores indicadores de salud que otras que no siguen esa misma línea, probablemente, debido a su menor margen financiero y autonomía fiscal.

Nuestros hallazgos apoyan así la hipótesis de asimetría federal entre tipos de regímenes por comunidades (Lopez-Casasnovas et al., 2005), los cuales deberían ser considerados a la hora de repensar la próxima reforma del sistema de financiación autonómico y garantizar igualmente mayores niveles de equidad pues el modelo vigente data ya de 2009. De igual forma, debieran hacernos reflexionar acerca de la efectividad real de las políticas sanitarias, según el contexto temporal y geográfico al cual se apliquen, tal y como se han venido orientando tradicionalmente hasta este momento.

Finalmente, es importante destacar las limitaciones del estudio. A pesar de que el modelo empleado nos permite tener una perspectiva general y extraer conclusiones para los objetivos planteados, para un mejor análisis de este tema en resultados en salud se necesitaría tanto considerar de algún modo el factor tecnológico (Thornton y Beilfuss, 2016) como poder utilizar micro datos. Esta extensión del estudio se deja para futuras investigaciones en las que habrá disponibles nuevos datos sobre indicadores de salud. 


\section{Referencias Bibliográficas}

Asociación de Economía de la Salud. (2013). Sistema Nacional de Salud: diagnóstico y propuestas de avance. Barcelona. Disponible en: http://www.aes.es/

Balgati, B.H. \& Moscone, F. (2010). Health care expenditure and income in the OECD reconsidered: evidence from panel data. Economic Modelling, 27, 804-811.

Barros, P.P. (1998). The black-box of health care expenditure growth determinants. Health Economics, 7, 533-544.

Blázquez, C., Cantarero. D. \& Pascual, M. (2014). Experiencia comparada europea y el reto de la descentralización sanitaria. Estudios de Economía Aplicada, 32(2), 841860.

Blázquez, C., González, N. \& Moreno, P. (2013). Pharmaceutical Expenditure as a Determinant of Health Outcomes in EU Countries. Estudios de Economía Aplicada, $50,31-2$.

Borrell, C., Peiró, R., Ramón, N., Pasarín, M. I., Colomer, C., Zafra, E. \& Álvarez-Dardet, C. (2005). Desigualdades socioeconómicas y planes de salud en las comunidades autónomas del Estado español. Gaceta Sanitaria, 19(4), 277-285.

Brown, M.C. (1987). Caring for profit: economic dimensions of Canada's health industry. Fraser Institute, Vancouver.

Cabases-Hita, J.M. (2006). La financiación del gasto sanitario desde la perspectiva de la experiencia comparada. Bilbao: Fundación BBVA.

Cabases-Hita, J.M. (2010). La financiación del gasto sanitario en España. Valoración del sistema de financiación, medida de la necesidad relativa y equidad. Fundación $B B V A$; 2010. Informe Economía y Sociedad.

Cantarero, D. (2006). Determinantes del gasto sanitario: un análisis empírico para el caso europeo. Estudios de Economía Española, 228, FEDEA, Madrid.

Cantarero, D. (2008). Desigualdades territoriales y equidad en el sistema sanitario español. Cuadernos económicos de ICE, 75, 141-166.

Cantarero, D., \& Lago-Peñas, S. (2010). The determinants of health care expenditure: a reexamination. Applied Economics Letters, 17(7), 723-726.

Cantarero, D. \& Lago-Peñas, S. (2012). Decomposing the determinants of health care expenditure: the case of Spain. The European Journal of Health Economics, 13(1), 19-27.

Cantarero, D. \& Pascual, M. (2008). Analysing the impact of fiscal decentralization on health outcomes: empirical evidence from Spain. Applied Economic Letters, (15), 109-111.

Costa-Font, J., \& Pons-Novell, J. (2007). Public health expenditure and spatial interactions in a decentralized national health system. Health Economics, 16(3), 291-306. 
Costa-Font, J. (2012). Fiscal Federalism and European Health System Decentralization: A Perspective. LEQS, Paper No. 55/2O12.

Cuckler, G. \& Sisko, A. (2013). Modeling per capita state health expenditure variation: state-level characteristics matter. Medicare \& Medicaid Research Review, 3(4), E1-E21.

De la Fuente, A. (2015). La evolución de la financiación de las comunidades autónomas de régimen común, 2002-2012. Papeles de Economía Española, 143, 28-49.

Di Matteo, L. (2003). The income elasticity of health care spending: a comparison of parametric and non-parametric approaches. European Journal of Health Economics, 4, 20-29.

Farag, M., Nandakumar, A.K., Wallack, S., Hodgkin, D., Gaumer, G. \& Erbil, C. (2012). The income elasticity of health care spending in developing and developed countries. International Journal of Health Care Finance and Economics, 12, 145-162.

Farag, M., Nandakumar, A.K., Wallack, S., Hodgkin, D., Gaumer, G. \& Erbil, C. (2013). Health expenditures, health outcomes and the role of good governance. International Journal of Health Care Finance and Economics, 13(1), 33-52.

García, F.P., Tormo, V.C., \& Lahiguera, L. H. (2015). Servicios públicos, diferencias territoriales e igualdad de oportunidades. Fundación BBVA.

Gerdtham, U.G., Jonsson, B., Macfarlan, M. \& Oxley, H. (1998). The determinants of health expenditure in the OECD countries. En Zweifel, P. (ed.): Health, The Medical Profession and Regulation, Kluwer Academic Publishers, Dordrecht.

Habibi, N., Huang, C., Miranda, D., Murillo, V., Ranis, G. \& Sarkar, M. (2003). Decentralization and Human Development in Argentina. Journal of Human Development, 4(1).

Jiménez-Rubio, D. (2011). The impact of decentralization of health services on health outcomes: evidence from Canada. Applied Economics, 43(26), 3907-3917.

Jiménez-Rubio, D. \& Smith, P. (2005). Decentralisation of health care and its impact on health outcomes. Discussion Papers in Economics, $N^{o} .2005 / 10$, University of York.

Kanavos, P. \& Mossialos, E. (1996). The methodology of international comparisons of health care expenditures: any lessons for health policy? LSE Health Discussion Paper, No. 3, London.

Kleiman, E. (1974). The determinants of national outlay on Health. Macmillan, London.

Labeaga, J. M. \& Ramiro, A. (2013). Rendición de cuentas con transparencia en el sector público: ċotra vez lo que el viento se llevó? Revista de Evaluación de Programas y Políticas Públicas, 1, 133-168.

Lago-Peñas, S., Cantarero-Prieto, D. \& Blázquez-Fernández, C. (2013). On the relationship between GDP and health care expenditure: a new look. Economic Modelling, 32, 124-129. 
Leu, R.E. (1986). The public-private mix and international health care costs. En Culyer, A.J., Jonsson, B. (Eds.): Public and Private health services, Basil Blackwell, Oxford, p. 41-63.

Lopez-Casasnovas, G., Costa-Font, J., \& Planas, I. (2005). Diversity and regional inequalities in the Spanish system of health care services'. Health EconomicsChichester, 14(1), S221.

López-Casasnovass, G. \& López-Valcárcel, B.G. (2015). El sistema sanitario en España, entre lo que no acaba de morir y lo que no termina de nacer. Papeles de Economía Española, en prensa.

Mahal, A., Srivastava, V. \& Sanan, D. (2000). Decentralization and its impact on public service provision on health and education sectors: the case of India, En: Dethier J (Ed.), Governance, Decentralization and Reform in China, India and Russia, Kluwer Academic Publishers and ZEF, London, p.77.

Newhouse, J.P. (1977). Medical care expenditure: a cross-national survey. Journal of Human Resources, 12, 115-125.

OECD Health Statistics (2015). http://www.oecd.org/els/health-systems/health-data. htm

Oliva, J., Ruiz-Giménez, J.L. \& Segura, J. (2014). Qué hacemos contra recortes y privatizaciones por un modelo universal y equitativo de la sanidad pública. Madrid: Akal.

Pérez, F. (dir.), Cucarella, V. \& Hernández, L. (2015). Servicios públicos, diferencias territoriales e igualación de oportunidades. Fundación BBVA, Madrid, p.248.

Pita-Barros, P. (2006). Gasto sanitario: ¿̇es más siempre mejor? Revista Asturiana de Economía, 35, 7-21.

Repullo, J.R. (2007). Gasto sanitario y descentralización: ¿saldrá a cuenta haber transferido el INSALUD? Presupuesto y Gasto Público, 49, 47-66.

Robalino, D.A., Picazo, O.F. \& Voetberg, A. (2001). Does fiscal decentralization improve health outcomes? Evidence from a cross-country analysis. Washington DC: World Bank, Policy Research Working Paper, 2565.

Sánchez, F.I., Abellán, J.M. \& Oliva, J. (2013). Gestión pública y gestión privada de servicios sanitarios públicos: más allá del ruido y la furia, una comparación internacional. Documentos de Trabajo 4/2013 del Real Instituto Elcano.

Sen, A. (2005). Is health care a luxury? New evidence from OECD Data. International Journal of Health Care Finance and Economics, 5, 147-164.

SESPAS. (2014). Crisis económica y salud. Informe SESPAS 2014. Gaceta Sanitaria, $28(S 1), 1-146$.

Thornton, J.A. \& Beilfuss, S.N. (2016). New evidence on factors affecting the level and growth of US health care spending. Applied Economics Letters, 23(1), 15-18. 
Urbanos, R. (2004). Tendencias internacionales en la financiación del gasto sanitario. Papeles de Trabajo del Instituto de Estudios Fiscales, 19.

Wendt, C. (2009). Mapping European healthcare systems: a comparative analysis of financing, service provision and access to healthcare. Journal of European Social Policy, 19, 432-445. 